\title{
Book report \\ Initial Management of Head Injury: A Comprehensive Guide
}

Claire Williams and David K Menon

Division of Anaesthesia, University of Cambridge, Box 93, Addenbrooke's Hospital, Cambridge CB2 2QQ, UK

Corresponding author: David K Menon, dkm13@wbic.cam.ac.uk

Published: 7 November 2008

Critical Care 2008, 12:310 (doi:10.1186/cc7104)

This article is online at http://ccforum.com/content/12/6/310

(c) 2008 BioMed Central Ltd

Selladurai B, Reilly P: Initial Management of Head Injury: A Comprehensive Guide. McGraw-Hill Medical; 2007, 315 pages. ISBN-10: 0074717758; ISBN-13: 978-0074717752.

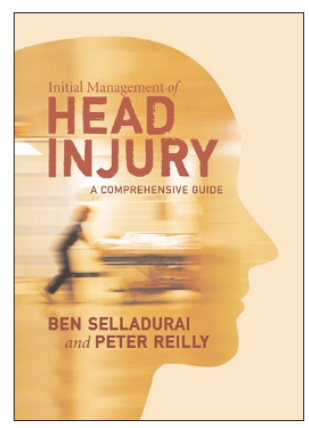

This book is aimed at the non-neurosurgeon involved in the immediate care of the head-injured patient. Written by neurosurgeons practicing in Malaysia and Australia, it draws heavily on North American and UK guidelines. The stated aim of the book is to provide advice to non-specialist clinicians, particularly in developing countries, who can use the evidencebased information in the book to develop local protocols.

The book contains six parts, covering epidemiology, basic principles, evaluation and diagnosis, immediate management, definitive management and management of special subsets of head-injured patients. Chapters are consistently arranged with an introduction, main body of information, summary and a comprehensive list of references. While logical, this means there is repetition and referring backwards and forwards within the book. The figures are, on the whole, simple and clear and illustrate the points that are being made. The layout, with multiple sections and subparagraphs and a somewhat arbitrary choice of fonts, is sometimes difficult to navigate.

The scope of the book extends beyond immediate management, but for a book such as this, less may be more, and future editions might benefit from judicious editing. Given the stated target readership of non-neurosurgeons, the detailed technical description of craniotomy is a bit unexpected but may be useful for the general surgeon who is forced to undertake emergency neurosurgery. The section on intracranial pressure measurement will be of more use to most emergency and intensive care unit physicians.

Areas covered particularly well involved the explanation of practical procedures. For example, the section on measuring and documenting the Glasgow Coma Scale score describes not only the scoring system, but also how scoring should be practically performed. Throughout, there is an emphasis on the neurological care of the patient, an aspect of management that is sometimes lost in the emergency room and during imaging and inter-hospital transfer of the patient.

However, there are problems in other areas, such as the recommendations for anaesthetic drug doses and ventilation parameters given in the section on immediate resuscitation. For example, in the chapter on 'Immediate management', the suggested tidal volumes of $15 \mathrm{~mL} / \mathrm{kg}$ are wholly out of keeping with any current management recommendations (and may actually be harmful). An incidental irritation is the implication in the accompanying highlighted text that hypoventilation causes hypocarbia. While this is a simple misprint, it might cause confusion to the inexperienced doctor dealing with an emergency in an isolated setting. If the book is to act as a primer for head injury management for such a clinical constituency, these details need to be accurate. Despite these caveats, this book will be of use to emergency physicians and intensive care unit, anaesthetic and surgical trainees who are regularly presented with head-injured patients but have limited access to specialist neurosurgical or neurocritical care advice.

\section{Competing interests}

The authors declare that they have no competing interests. 